\title{
Encefalopatías espongiformes transmisibles
}

Jorge E D elgado-Hachmeister, MC, (1) M Sigfrido Rangel-Frausto, MC, ${ }^{(2)}$ Samuel Ponce de León, MC. ${ }^{(3)}$

\begin{abstract}
Delgado-Hachmeister JE, Rangel-Frausto MS, Ponce de León S. Encefalopatías espongiformes transmisibles. Salud Publica Mex 2002;44:69-75. El texto completo en inglés de este artículo está disponible en: http://www.insp.mx/salud/index.html

\section{Resumen}

Las encefalopatías espongiformes transmisibles (EET) han cobrado gran importancia en los últimos años. Principalmente por el surgimiento de la encefalopatía espongiforme del bovino (EEB) y la nueva variante de la enfermedad de C reutzfeldt-Jakob (nvECJ), esta última probablemente adquirida por la ingesta de carne de bovino contaminada. Hasta la fecha se ha informado de 109 casos de la nvEC] en el humano y la gran mayoría de los casos ha ocurrido en el Reino U nido. N o se sabe la magnitud real que podrán tener las EET en el humano, sin embargo algunos piensan que nos encontramos en el principio de una pandemia de la nvECJ. En el presente artículo se discuten varios aspectos de las EET y métodos para la prevención de la transmisión de estas enfermedades, tanto en rumiantes como en el humano. El texto completo en inglés de este artículo está disponible en: http://www.insp.mx/salud/index.html
\end{abstract}

Palabras clave: encefalo patías espongiformes transmisibles; encefalopatía espongiforme bovina; enfermedad de c reutzfeldt-Jakob; infección hospitalaria

\section{Delgado-Hachmeister JE, Rangel-Frausto MS, Ponce de León S. Transmissible spongiform encephalopathies. Salud Publica Mex 2002;44:69-75. \\ The English version of this paper is available at: http://www.insp.mx/salud/index.html}

\section{Abstract}

Transmissible spongiform encephalopathies (TSE) are a group of diseases which have received a lot of attention in recent years. The interest on these diseases has been stimulated by the appearance of bovine spongiform encephalopathy (BSE) and the new variant of Creutzfeldt-Jakob disease (nVC JD); the latter is likely to be acquired by ingesting contaminated beef. Until now 109 cases of nVCJD have been reported, most of them occurring in the U nited Kingdom. Some experts think that this is the beginning of a nvC JD pandemic. Deep knowledge of the mechanisms of transmission of TSE is needed to prevent the emergence of a TSE pandemic in humans. We address various aspects ofTSE and discuss prevention methods of TSE in ruminants and humans. The English version of this paper is available at: http:/ /www.insp.mx/salud/index.html

Key words: transmissible spongiform encephalopathies; encephalopathy, bovine spongiform; Creutzfeldt-Jakob disease; cross infection as encefalopatías espongiformes transmisibles (EET), o enfermedades relacionadas con priones, son un grupo de condiciones neurológicas, estrechamente relacionadas por sus características histopatológicas, que ocurren en humanos y animales. Las EET se desarrollan por mecanismos hereditarios, esporádicos o infecciosos. En años recientes las enfermedades relacionadas con priones han generado mucho interés, sobre todo por la epidemia de encefalopatía espongiforme del bovino (EEB) y el surgimiento de la nue-

(1) Universidad la Salle, Facultad Mexicana de Medicina, México, D.F., México.

(2) Unidad de Investigación Médica en Epidemiología Hospitalaria, Centro Médico N acional Siglo XXI, Instituto Mexicano del Seguro Social, México, D.F., México.

(3) Subdirección de Epidemio logía H ospitalaria y Control de Calidad de laAtención Médica. Instituto N acional de Ciencias Médicas y N utrición Salvador Zubirán, México, D.F., México.

Fecha de recibido: 8 de mayo de 2001 - Fecha de aprobado: 20 de agosto de 2001 Solicitud de sobretiros: Dr. Samuel Ponce de León.Vasco de Q uiroga 15, Delegación Tlalpan, 14000 México, D.F. Correo electrónico: sponce@ quetzal.innsz.mx 
va variante de la enfermedad de Creutzfeldt-Jakob (nvECJ), esta última aparentemente relacionada con la ingesta de alimentos provenientes de vacas con EEB. ${ }^{1,2}$

\section{Enfermedades relacionadas con priones en animales}

La EET de ovejas y cabras (scrapie) es la enfermedad relacionada con priones prototípica. Esta se conoce desde hace más de 250 años como una enfermedad enzoótica. En el Reino Unido de 0.5 a 1\% de las ovejas presentan esta condición cada año.

La EEB fue descubierta en 1985 en el Reino Unido, y ha evolucionado de forma epidémica calculándose que hasta un millón de vacas fueron infectadas con la EEB en esta zona. También se han documentado diversos casos de EEB en otros países europeos, aunque limitados, y hasta ahora con una dimensión menor a la observada en el Reino Unido, pero los casos aumentan año con año. Tan sólo del 1 de enero al 6 de agosto de 2001 se han reportado 87 casos de EEB en Alemania, y en el mismo periodo se informan ya 52 en España. ${ }^{3}$ Estos datos son preocupantes, ya que en años anteriores la ocurrencia de casos de EEB en otros países europeos no ocurría o era un fenómeno raro. En el continente americano, incluyendo México, no se han informado casos autóctonos deEEB. ${ }^{3}$

El origen de la EEB probablemente proviene de alimento para ganado vacuno manufacturado a partir de rumiantes (ovejas y vacas) con EET, principalmente carne y harinas de hueso. ${ }^{4,5}$ Debido a que en el Reino Unido la EET de ovejas y cabras es endémica, se plantea la hipótesis de que los desechos de ovejas contaminados fueron la fuente inicial de la EEB. Sin embargo, también se postula que los priones de la EEB tuvieron su origen de manera espontánea en el ganado vacuno, de tal manera que la enfermedad fue amplificada por el reciclamiento de desechos de vacas.

A pesar de que no se han informado casos de EEB en México, es necesario mantener una alerta epidemiológica para evitar una epidemia similar a la que se observa en Europa.

El diagnóstico clínico de esta enfermedad en los bovinos se sospecha por la aparición de diversos síntomas y signos. Entre los síntomas encontramos: aprehensión, miedo, mirada fija frecuente o depresión. De los signos se distinguen hiperestesia o hiperreflexia, fasciculaciones musculares, temblor y mioclonía, ataxia en la marcha, incluyendo hipermetría, y disfunción autonómica, como rumiación disminuida, bradicardia y un ritmo cardiaco alterado. ${ }^{3}$ En el caso de que se sospeche EEB se debe hacer un estudio detallado del cerebro y del tallo cerebral después de la muerte del animal, tan rápido como sea posible.
Para prevenir el surgimiento de esta enfermedad en México, es importante que se realicen estudios histopatológicos de vigilancia en rumiantes con enfermedad neurológica; imponer restricciones sobre la importación de especies vivas de rumiantes y sus productos; diseñar una política para la importación de embriones; y la prohibición del enriquecimiento del alimento con cadáveres del mismo género (rumiantes con rumiantes). Cuando se comuniquen casos de EEB, no se debe penalizar a los ganaderos, sino ofrecer compensación a los mismos, con el objeto de disminuir el subregistro e impedir que animales enfermos entren a la cadena alimenticia humana.

\section{Enfermedades relacionadas con priones en humanos}

Las enfermedades relacionadas con priones en humanos son únicas en la biología, ya que tienen etiología esporádica, genética o infecciosa. Se han descrito diferentes variantes de enfermedades relacionadas con priones en humanos, que incluyen Kuru, Enfermedad de Creutzfeldt-Jakob (ECJ) familiar, ECJ esporádica, ECJ iatrogénica y la nueva variante de la ECJ, síndrome de Gerstmann-Sträussler-Scheinker, insomnio fatal familiar e insomnio fatal espontáneo. ${ }^{6}$

La mayoría de casos de las enfermedades relacionadas con priones ocurren espontáneamente, tal es el caso de la ECJ esporádica (ECJe) que sucede en una persona de cada un millón de habitantes. La distribución de la ECJe es igual en hombres y mujeres. La etiología de la ECJe no se conoce, se postula que hay una mutación somática del gene $\operatorname{PrP}$ (Proteína de priones); ésta ocurre como un fenómeno estocástico raro, la proteína $\mathrm{PrPc}^{\mathrm{c}}$ cambia a $\operatorname{PrP}^{\mathrm{sc}}$. Existe un polimorfismo común y benigno en el codón 129 del gene PrP que codifica para metionina o valina. Los homocigotos para metionina en esta posición tienen un mayor riego para desarrollar ECJ esporádica o iatrogénica. ${ }^{7}$

Existen tres hipótesis acerca de la naturaleza del agente infeccioso de las EET. La primera postula que estas enfermedades son causadas por una proteína que se replica a sí misma. ${ }^{8}$ La segunda plantea que el agente es un virus pequeño. ${ }^{9}$ La tercera dice que se trata de un virino. Un virino es una molécula pequeña (posiblemente un ácido nucleico) asociado con una proteína huésped, tal vez PrP. ${ }^{10}$ Hasta la fecha la que más credibilidad goza es la hipótesis proteica de la enfermedad. Esta cobró fuerza con el otorgamiento del premio Nobel de Medicina a Stanley Prusiner en 1997. ${ }^{11}$ Es la primera ocasión en que este galardón se ofrece a un trabajo todavía hipotético y no totalmente comprobado. Discutiremos brevemente la hipótesis proteica. De 
acuerdo con esta hipótesis los priones son partículas transmisibles que no poseen ácidos nucleicos y que están compuestos exclusivamente de una proteína modificada $\operatorname{PrP}^{\text {sc }}$. La forma normal celular, $\mathrm{PrP}^{\mathrm{Pc}}$, es convertida a PrPsc a través de un procesamiento postraduccional, por medio del cual la PrP adquiere un contenido alto de hélices $b .{ }^{11-13}$

A pesar de que las enfermedades relacionadas con priones humanos se pueden transmitir de manera experimental, las formas adquiridas hasta hace poco habían estado confinadas a situaciones inusuales. Por ejemplo, el kuru fue diseminado por el canibalismo ritual en Papua, Nueva Guinea. ${ }^{14}$ Otros casos de enfermedades humanas relacionadas con priones adquiridas han resultado de la transmisión iatrogénica de ECJ.

Esta transmisión ha ocurrido durante el transplante de córneas, colocación de injertos de dura madre, administración de hormona de crecimiento humana cadavérica, implantación de electrodos electroencefalográficos intracraneanos contaminados y cirugías (sobre todo neurocirugía y cirugía oftalmológica) en donde se utilizan instrumental o aparatos quirúrgicos contaminados. ${ }^{15}$

\section{La nueva variante}

En 1994 se presentaron en la Gran Bretaña, por primera vez, casos de ECJ en adolescentes y adultos jóvenes, y recibieron eventualmente el nombre de nueva variante de $\mathrm{ECJ}(\mathrm{nvECJ}) .{ }^{16}$ Hasta entonces era extremadamente raro que se presentaran casos de ECJ en gente joven y además las características histopatológicas eran inusuales; había presencia de placas de amiloide formadas con $\mathrm{PrP}$ (proteína de priones) rodeadas por un halo de degeneración espongiforme intenso. El surgimiento de casos de nvECJ en una zona geográfica restringida (principalmente Reino Unido), en la que simultáneamente ocurre la epidemia de EEB, ha hecho pensar que la EEB cruzó la barrera interespecie y que fue transmitida a humanos por carne de vaca contaminada.

Hasta el 6 de agosto de 2001 se han informado en el Reino Unido 106 casos definitivos o probables de la nvECJ. Destaca que en el año 2000 se diagnosticaron 28 casos definitivos, que representa el número más alto informado desde 1995, además la tendencia general, desde el inicio de la epidemia en el Reino Unido, muestra que año con año se presentan más casos de la nvECJ. ${ }^{17}$ En Francia se han diagnosticado dos pacientes con la nvECJ y en Irlanda uno.

Los estudios epidemiológicos no han identificado hábitos dietéticos distintos en los sujetos afectados y no se sabe por qué la nvECJ afecta preferentemente a adolescentes y adultos jóvenes. Realmente no cono- cemos si estamos en el comienzo de una epidemia de enfermedad relacionada con priones en humanos, similar a lo sucedido con el kuru o con la EEB. Algunos calculan que cientos de miles de personas desarrollarán la nvECJ, mientras que otros postulan que los casos de la nvECJ van a ser pocos, similar a lo ocurrido con la ECJ iatrogénica. ${ }^{18,19}$

Nosotros sugerimos que se tomen todas las precauciones necesarias, incluyendo las que discutimos en este artículo, para prevenir el desarrollo de casos de EEB y de la nvECJ en México. El no adoptar las precauciones que discutimos puede ser una difícil lección en años venideros.

La nvECJ se caracteriza por el desarrollo de un trastorno neuropsiquiátrico progresivo que se manifiesta por la aparición de ataxia, demencia, mioclonía o corea, sin el patrón típico electroencefalográfico (EEG) de la ECJe. Por otro lado, como ya mencionamos, existe un patrón histopatológico distinto. Por lo tanto, para poder confirmar un caso de la nvECJ se requiere efectuar un estudio histopatológico cerebral.

En el cuadro I enumeramos los criterios diagnósticos para la nvECJ.

\section{Cuadro I \\ Criterios diagnósticos para la nueva variante de la enfermedad de Creutzfeldt-Jakob}

A. Trastorno neuropsiquiátrico progresivo

IB. Duración de la enfermedad $>6$ meses

IC. No existe otra alternativa diagnóstica

ID. N o existe historia de exposición iatrogénica potencial

IIA. Síntomas psiquiátricos tempranos (depresión, ansiedad, apatía, aislamiento y delirio)

IIB. Presencia de síntomas sensitivos persistentes (incluye dolor franco y disestesia)

IIC.A taxia

IID. Mioclonía, corea o distonía

IIE. Demencia

III

IIIA. Electro encefalograma sin alteraciones típicas de la ECJ esporádica* IIIB. Imagen de Resonancia Magnética con áreas hiperintensas bilaterales en región pulvinar

IV

IVA. Biopsia amigdalina positiva

*Complejos periódicos trifásicos generalizados con frecuencia de $1 \mathrm{~Hz}$

El diagnóstico es definitivo si cumple con el punto IA y además se confirma neuropatológicamente la presencia de la nvEC) (C ambio espongiforme y depósitos amplios de PrP con placas floridas, a lo largo del cerebro y cerebelo). El diagnóstico es probable si cumple con el punto I completo, 4 de 5 criterios del inciso II, IIIA y IIIB o si cumple con el criterio I y IVA.

Consulte http://www.doh.gov.uk/cjd/stats/aug01.htm ${ }^{17}$ 


\section{Guías para la prevención de la transmisión de las EET en el hospital}

Las siguientes guías están basadas, casi en su totalidad, en un documento publicado por la organización mundial de la salud. ${ }^{20}$

Considerando que las EET pueden ser adquiridas iatrogénicamente se han diseñado estrategias para prevenir su transmisión. Algunas de estas recomendaciones son poco prácticas en ocasiones. Sin embargo, el personal médico que se ve involucrado en el manejo de pacientes con EET debe seguirlas hasta donde sea posible. A ningún paciente con EET se le debe negar cualquier procedimiento, ya que si se siguen las recomendaciones propuestas el riesgo de transmisión a otros pacientes o trabajadores de la salud es mínimo.

Los agentes de las EET son inusualmente resistentes a métodos químicos y físicos de descontaminación. No son inactivados por la gran mayoría de los desinfectantes, o fijadores de tejido, además puede persistir cierta infectividad residual bajo condiciones de autoclave estándar (p.ej.: $121^{\circ} \mathrm{C}$ por 15 minutos). Son extremadamente resistentes a dosis altas de radiación ionizante y ultravioleta. Por otro lado, se ha demostrado que los agentes de la EET pueden sobrevivir por largo tiempo en el medio ambiente.

\section{Transmisión iatrogénica}

Las EET no son transmitidas de persona a persona, pero teóricamente esto puede ocurrir durante la práctica de procedimientos médicos invasivos. Para prevenir la transmisión de EET de pacientes a otros individuos (pacientes y trabajadores de la salud) es conveniente estratificar para distintas categorías de riesgo. Este riesgo depende de tres consideraciones: 1. La probabilidad de que un individuo desarrolle una EET; 2 . El grado de infectividad de los tejidos o líquidos de estos pacientes; 3 . La naturaleza y ruta de exposición a estos tejidos.

Los pacientes con más alto riesgo son aquellos con el diagnóstico o la sospecha de EET. La información disponible indica que los tejidos con más alta infectividad son los del Sistema Nervioso Central (cerebro, médula espinal y ojo). Los tejidos con baja infectividad están representados por el líquido cerebroespinal y varios otros órganos fuera del SNC (pulmón, hígado, riñon, bazo/ganglios linfáticos y placenta). Los tejidos en los que nunca se ha detectado infectividad incluyen el corazón, músculo esquelético, nervios periféricos, tejido adiposo, tejido gingival, intestino, glándulas suprarrenales, tiroides, próstata, testículos y secreciones corporales (orina, heces, saliva, moco, semen, leche, lágrimas, sudor y exudado seroso).
Cuando se calcula el riesgo debemos también considerar la forma de exposición. La exposición de la piel y mucosas intactas, a excepción de la del ojo, implica un riesgo casi nulo; sin embargo, es prudente evitar tal tipo de contacto, sobre todo si se trabaja con tejidos de alta infectividad. Las exposiciones transdérmicas, incluyendo exposición de la piel y mucosas no intactas, salpicaduras sobre los ojos y las heridas infringidas por instrumentos punzocortantes (por ejemplo agujas) imponen un riesgo potencial mayor. Se recomienda evitar tal tipo de exposición cuando se trabaja con tejidos de alta o baja infectividad. La exposición del SNC (por ejemplo inoculación del ojo o SNC ) con cualquier tipo de material infeccioso representa un riesgo muy serio y se debe implementar siempre las precauciones necesarias para evitar este tipo de exposición.

\section{Cuidado del paciente}

El contacto social habitual y los procedimientos diagnósticos no invasivos no implican ningún tipo de riesgo para los trabajadores de la salud, sus parientes o la comunidad. Los pacientes con EET no se deben aislar, pueden estar en cuartos no privados, sólo se recomienda apegarse a las precauciones estándar. Sin embargo, debido a que las EET progresan rápidamente, el paciente pronto desarrolla dependencia por lo que la asignación de una enfermera especial, en un cuarto privado, es recomendable por razones humanitarias.

Las excretas del paciente se deben manejar de acuerdo con las leyes municipales. No se requieren precauciones especiales para cubiertos, sondas de alimentación y succión, y sábanas.

\section{Procedimientos dentales}

No existe evidencia epidemiológica que indique que los procedimientos dentales se asocian con la adquisición iatrogénica de una EET. Sin embargo, estudios en animales indican que el tejido gingival y la pulpa dental son potencialmente infecciosos. Y por ello se recomienda utilizar precauciones especiales en pacientes con EET cuando se realicen procedimientos que involucren tejido neurovascular (por ejemplo endodoncia). En intervenciones dentales, donde no se trabaje con el tejido neurovascular, los métodos preventivos habituales son suficientes. Las precauciones incluyen utilizar instrumentos desechables; incinerar el equipo dental contaminado con tejido neurovascular o descontaminarlo por un método efectivo que elimine a los agentes de las EET (ver abajo); y programar los procedimientos al final del día para poder limpiar adecuadamente los instrumentos. ${ }^{20}$ 
Procedimientos diagnósticos

A los pacientes con EET, al igual que a otros pacientes, se les practican cierto tipo de procedimientos diagnósticos que incluyen oftalmoscopia, endoscopia, colonoscopia, colocación de catéteres urinarios o vasculares y pruebas de función pulmonar o cardiaca. ${ }^{20}$

En este tipo de procedimientos no se debe tomar ninguna precaución especial. Pero se sugiere programar estas intervenciones diagnósticas al final del día, de tal manera que los instrumentos se limpien más cuidadosamente. Sin embargo, cuando los instrumentos se exponen a tejidos de alta o baja infectividad (ver arriba), estos se deben someter al proceso de esterilización más intenso que puedan tolerar ${ }^{20}$ (ver abajo).

\section{Transfusiones}

Distintos países han diseñado políticas para disminuir el riesgo de transmisión de EET por medio de productos sanguíneos. Teóricamente puede haber transmisión por hemoderivados de EET, incluso por pacientes aparentemente sanos pero que se encuentran en la fase de incubación de la enfermedad. Estudios experimentales indican que la transmisión de EET de oveja a oveja, durante la fase asintomática de la enfermedad, es posible por medio de transfusiones sanguíneas. ${ }^{21}$

En los Estados Unidos de América la política de donadores excluye a personas que estuvieron en el Reino Unido por un tiempo acumulativo igual o mayor a seis meses, de 1980 hasta 1996.22 Otros países han adoptado esta medida, entre los que se incluyen Canadá, Australia, Nueva Zelanda, Suiza, Japón y Alemania. Esta práctica también se debe aplicar en nuestro país hasta que exista evidencia suficiente que descarte la posibilidad de transmisión de las EET por transfusión de productos sanguíneos.

\section{Procedimientos quirúrgicos}

Cuando un paciente con sospecha o diagnóstico de EET, programado para cirugía, ingresa al hospital, la división encargada del control de infecciones hospitalarias debe ser informada al respecto. Entonces se planea de manera cuidadosa, por un grupo interdisciplinario (cirujano, central de esterilización, epidemiólogo hospitalario, infectólogo, etcétera) el procedimiento, y el manejo, almacenamiento, limpieza, descontaminación y desecho de los instrumentos utilizados durante la cirugía; de tal manera que la intervención se haga en una sala de operaciones; involucre a un equipo compacto de personal quirúrgico; se utilice equipo de un solo uso (batas quirúrgicas, guantes, máscaras, visor, campos quirúrgicos); se marque todo el equipo reutilizable; se mantenga el flujo de instrumentos en una sola línea; se incineren todos los desechos e instrumental quirúrgico (cuando esto sea posible); se marquen todas las muestras como residuos biológico infecciosos, y se limpien todas las superficies de acuerdo con recomendaciones establecidas ${ }^{20}$ (ver abajo).

\section{Manejo del instrumental quirúrgico}

Los métodos para descontaminación del instrumental quirúrgico se describen más adelante. El método se determina por el grado de infectividad de los tejidos y la forma en la cual los instrumentos van a ser posteriormente reutilizados. En caso de que los utensilios quirúrgicos entren en contacto con tejidos de alta infectividad se recomienda el uso de instrumentos de un solo uso. En el caso de que no se cuente con instrumentos de un solo uso, lo más adecuado es destruir estos instrumentos reutilizables.

A pesar de que el líquido cerebroespinal se clasifica como tejido de baja infectividad se recomienda que los instrumentos que estuvieron en contacto con él se manejen de la misma manera que si se tratara de instrumental contaminado con tejidos de alta infectividad. Por supuesto que esto se aplica al instrumental que se usa para una punción lumbar.

El instrumental quirúrgico utilizado en tejidos de alta o baja infectividad, se debe colocar en un contenedor robusto y a prueba de fugas, que tenga la etiqueta "residuos biológico infecciosos". Este instrumental debe ser transferido al departamento de esterilización y ser esterilizado con un método efectivo (ver abajo), o ser transferido al incinerador. Esto debe suceder de una manera rápida y eficiente. ${ }^{20}$

\section{Cuarentena}

En caso de que se sospeche del diagnóstico de una EET, si los instrumentos se pueden guardar en un lugar seguro, estos se pueden esterilizar con el mejor método posible y guardar en contenedores rígidos, hasta que se confirme o descarte el diagnóstico de EET.

\section{Procedimientos de descontaminación}

Los agentes de la EET son inusualmente resistentes a métodos de esterilización y desinfección tradicionales. La variabilidad en la efectividad de los métodos de descontaminación se ve influenciada por la naturaleza y estado físico de los tejidos infectados. Se sabe, por ejemplo, que la infectividad es estabilizada por métodos de fijación como alcohol, formalina o glutaraldehido o 
cuando las superficies se secan. Por lo tanto, los materiales contaminados no se deben exponer a sustancias de fijación y se deben mantener sumergidos en desinfectantes químicos desde que se utilizan hasta que son desinfectados.

El método más seguro para disminuir el potencial de infectividad residual es que el instrumental quirúrgico sea incinerado. Sin embargo, esto no es posible con todo el material quirúrgico. En este caso se debe utilizar el proceso de descontaminación más efectivo que pueda tolerar el instrumento (ver abajo).

Cuando sea posible se deben combinar dos métodos de desinfección efectivos para el instrumento. Los procedimientos que utilizan calor y $\mathrm{NaOH}$ (ya sea simultánea o consecutivamente) parecen ser los más efectivos.

Algunos instrumentos costosos, como endoscopios fibroópticos, no pueden ser sometidos a procedimientos tan intensos de descontaminación. Estos se pueden proteger envolviéndolos de tal manera que se disminuya la exposición a superficies contaminadas.

\section{Métodos de descontaminación para encefalopatías espongiformes transmisibles}

El método más efectivo en reducir el riesgo de infectividad residual es la destrucción de los instrumentos por medio de incineración. Cuando sea posible, los instrumentos y otros materiales sujetos a reutilización, se deben mantener húmedos desde el momento de la exposición hasta la descontaminación. En caso de que se pueda realizar de una manera segura, se deben remover partículas adheridas a los instrumentos, por medio de una limpieza de tipo mecánica.

Las siguientes recomendaciones enumeran los métodos de desinfección recomendados, en orden de efectividad. Estas recomendaciones se basan en el reporte de la Organización Mundial de la Salud. Nuevos métodos pueden surgir conforme se acumula más evidencia científica.

\section{Incineración}

- Utilice para todos los instrumentos, materiales y basura desechable

- Método de elección para todos los instrumentos expuestos a tejidos de alta infectividad.

2. Autoclave / Métodos químicos para instrumentos resistentes a calor

- Sumerja en hidróxido de sodio $(\mathrm{NaOH})$ y caliente en un autoclave de desplazamiento de gravedad a $121^{\circ} \mathrm{C}$ por 30 minutos; limpie; lave en agua y someta a métodos de esterilización de rutina

- Sumerja en $\mathrm{NaOH}$ o hipoclorito de sodio por una hora; transfiera los instrumentos a agua; caliente en un autoclave de desplazamiento de gravedad a $121^{\circ} \mathrm{C}$ por una hora; limpie y someta a esterilización de rutina

- Sumerja en $\mathrm{NaOH}$ o hipoclorito de sodio por una hora; lave en agua y después transfiera a un recipiente abierto y caliente en un autoclave de desplazamiento de gravedad $\left(121^{\circ} \mathrm{C}\right)$ o de carga porosa $\left(134^{\circ} \mathrm{C}\right)$ por una hora; limpie y someta a esterilización de rutina

- Sumerja en $\mathrm{NaOH}$ y hierva por 10 minutos a presión atmosférica; limpie, lave en agua y someta a esterilización de rutina

- Sumerja en hipoclorito de sodio (preferido) o $\mathrm{NaOH}$ (alternativo) a temperatura ambiente por una hora; limpie; lave en agua y someta a esterilización rutinaria

- Autoclave a $134^{\circ} \mathrm{C}$ por 18 minutos.

3. Métodos químicos para superficies e instrumentos sensibles a calor

- Lave con $\mathrm{NaOH}(2 \mathrm{~N})$ o hipoclorito de sodio no diluido y sumérjalo por una hora; retire y enjuague con agua

- En caso de que las superficies no toleren la exposición a $\mathrm{NaOH}$ o el hipoclorito, un lavado extenso remueve la gran mayoría de la infectividad por dilución, además se puede obtener una mayor desinfección al lavar con un método parcialmente efectivo.

\section{Conclusiones}

La epidemia europea de EEB es preocupante; año con año se reportan más casos de EEB en diversos países europeos. Esta información nos hace pensar que se puede presentar un fenómeno similar al ocurrido en el Reino Unido en otros países del mundo: una epidemia de gran magnitud de EEB y finalmente la ocurrencia de casos de la nvECJ por transmisión interespecie de priones por carne contaminada de ganado vacuno a humanos. Hasta la fecha no sabemos cuál va a ser la magnitud de la nvECJ, sin embargo es posible que se presenten miles de casos de la misma en años venideros. En países del continente americano, incluyendo México, no ha habido reportes de EEB, no obstante es sumamente importante que establezcamos las medidas necesarias para prevenir la emergencia de esta enfermedad en nuestro ganado, éstas deben incluir, pero no se limitan 
a la prohibición de la importación de especies vivas, sus productos y embriones de rumiantes de la gran mayoría, si no es que todos, los países europeos, y la prohibición del enriquecimiento del alimento con cadáveres del mismo género. El no seguir estas recomendaciones puede ser una difícil lección para nuestra industria ganadera y finalmente para muchos mexicanos que podríamos desarrollar la nvECJ. El surgimiento de las EET en humanos, además, hace necesario que todos los trabajadores de la salud conozcamos los mecanismos de transmisión, el cuadro clínico y las medidas necesarias que se deben seguir para prevenir la transmisión nosocomial de las EET. Si bien el número de casos reportados de la nvECJ es limitado, existe la posibilidad de que se trate de una enfermedad de gran magnitud con características de gran preocupación: afecta gente joven, produce degeneración del sistema nervioso central, es progresiva, rápidamente mortal y no existe tratamiento disponible.

\section{Referencias}

1. Zeidler M, Stewart GE, Barraclough CR, Bateman DE, Bates D, Burn DJ et al. $\mathrm{N}$ ew variant $\mathrm{Creutzfeldt-Jakob}$ disease: $\mathrm{N}$ eurological features and diagnostic tests. Lancet 1997;350:903-907.

2. Collinge J, Sidle KCL, Meads J, Ironsidce J, Hill AF. Molecular analysis of prion strain variation and the aetiology of 'new variant' CJD. Nature 1996;383:685-690.

3. 0 ffice International des Epizooties. 0 rganisation mondiale de la santé animale. $N$ umber of reported cases of bovine spongiform encephalopathy (BSE) world wide (excluding the United Kingdom). Switzerland. U pdated 06.08.2001. Disponible en: http://www.oie.int/info/en_esbmonde.htm 4. Prusiner SB. Prion diseases and the BSE crisis. Science 1997;278:245251.

5. Prusiner SB.The prion diseases. Sci Am 1995;268:48-57.
6. Telling GC, Collinge J. Transmissible spongiform encephalopathies of humans and animals. En: Infect Dis. Armstrong D, ed. Essex Mosby 1999: 19.1-19.6

7. Collinge J, Palmer MS, D ryden AJ. G enetic predisposition to iatrogenic Creutzfeldt-Jakob disease. Lancet 1991;337:1441-1442.

8.W eissmann C.The ninth datta lecture: Molecular biology of transmissible spongiform encephalopathies. FEBS Lett 1996;389:3-11.

9. Manuelidis L.The dimensions of C reutzfeldt-Jakob disease. Transfusion 1994;34:915-928.

10. Dickinson AG, 0 utram GW. Genetic aspects of unconventional virus infections:The basis of the virino hypothesis. Ciba Found Symp 1988;135:6383.

11. Prusiner SB. N obel lecture: Prions. Proc N atl A cad Sci 1998:95:1336313383.

12. Haywood AM. Transmissible spongiform encephalopathies. N Engl J Med 1997;337:1821-1828.

13. Prusiner SB. Prions and neurodegenerative diseases. N Engl J Med 1987;317:1571-1581.

14. Gajdusek DC. Unconventional viruses and the origin and disappearance of kuru. Science 1977;197:943-960.

15. Brown P, Preece MA,W ill RG. "Friendly fire" in medicine: Hormones, homografts, and Creutzfeldt-Jakob disease. Lancet 1992;340:24-27.

16.W ill RG, Ironside JW, Zeidler M, Cousens SN , Estibeiro K,A A perovitch $A$ et al. A new variant of Creutzfeldt-Jakob disease in the UK. Lancet 1996;347:921-925

17. D epartment of Health. Monthly C reutzfeldt-Jakob D isease Statistics. 2001/0369. Monday $6^{\text {th }}$ A ugust 2001. U nited Kingdom. D isponible en: http:/ /www.doh.gov.uk/cjd/stats/aug01.htm.

18. G hani AC, Ferguson N M, D onnelly CA,Anderson RM. Predicted VC JD mortality in Great Britain. $N$ ature 2000;406:583-584.

19. Cousens SN ,Vynnycky E, Zeidler M,W ill RG, Smith PG. Predicting the CJD epidemic in humans. Nature 1997;385:197-198.

20. W HO infection control guidelines for transmissible spongiform encephalo pathies: Report of a W HO Consultation. Ginebra:W HO /CDS/ CSR/APH/2000.3, March 23-26, 1999. D isponible en: http://www.who.int/ emc/diseases/bse/.

21. Houston F, Foster JD, C hong A, Hunter N, Bostock CJ.Transmission of BSE by blood transfusion in sheep. Lancet 2000;356:999-1000.

22. Brown P,W ill RG, Bradley R,A sher DM, D etwiler L. Bovine spongiform encephalopathy and variant Creutzfeldt-Jakob disease: Background, evolution, and current concerns. Emerg Infect D is 2001;7:6-16. 